Squeeze Me and l'll Change: An Exploration of Frustration-triggered Adaptation for Multimodal Interaction

Peer-reviewed author version

OCTAVIA, Johanna; CONINX, Karin \& LUYTEN, Kris (2011) Squeeze Me and I'Il Change: An Exploration of Frustration-triggered Adaptation for Multimodal Interaction. In: Proceedngs of the IEEE Symposium on 3D User Interfaces 2011. p. 79-86..

Handle: http://hdl.handle.net/1942/12150 


\title{
Squeeze Me and I'll Change: An Exploration of Frustration-triggered Adaptation for Multimodal Interaction
}

\author{
Johanna Renny Octavia* Karin Coninx $x^{\dagger}$ \\ Hasselt University - tUL - IBBT \\ Expertise Centre for Digital Media
}

\begin{abstract}
Complex 3D interaction in virtual environments may inhibit user interaction and cause frustration. Supporting adaptivity based on the detected user frustration can be considered as one promising solution to enhance user interaction. Our work proposes to provide adaptive assistance to users who are frustrated during their interaction with 3D user interfaces in virtual environments. The obtrusiveness of physiological measurements to detect frustration inspired us to investigate the pressure patterns exerted on a 3D input device for this purpose. The experiment presented in this paper has shown a great potential on utilizing the finger pressure measures as an alternative to physiological measures to indicate user frustration during interaction. Furthermore, the findings in this particular context showed that adaptation of haptic interaction was effective in increasing the user's performance and making users feel less frustrated in performing their tasks in the 3D environment.
\end{abstract}

Index Terms: I.3.7 [Three-Dimensional Graphics and Realism]: Virtual reality; H.5.2 [User Interfaces]: Input devices and strategies; B.4.2 [Input/Output Devices]: Channels and controllers

\section{INTRODUCTION}

Despite the fact that the development of three-dimensional user interfaces has matured and is backed by over forty years of 3D technology research, ensuring usability for this class of user interfaces remains cumbersome. 3D interaction is supposed to be more intuitive and natural - because it resembles real life - but studies show this is highly dependent on the user [1]. There is still a large number of users that consider understanding 3D spaces and performing actions in virtual environments to be inherently difficult, due to the complex, highly-interactive tasks and the amount of different 3D interaction techniques and devices. This may hinder user interaction, cause user frustration and in the end also affect user performance.

Frustration is a universal experience for computer users, and for many users frustrating experiences occur on a frequent basis during their interaction. It was observed that due to frustrating experiences, the time lost ranged from 47 to $53 \%$ of the total time users spent on a computer [3] during the use of applications such as web browsing, e-mail, and word processing. Owing to frustration, users may suffer a significant decrease in their performance. According to Norman's interaction framework [19], the sources of difficulties in user interaction are the gulf of execution and the gulf of evaluation. The larger these gulfs are, the more easily the user becomes frustrated. Users experience frustration when there is an inhibiting condition which hinders the completion of an action or task. In virtual environments, we presumably think that the nature of complex

\footnotetext{
*e-mail: johanna.octavia@uhasselt.be

†e-mail:karin.coninx@uhasselt.be

‡e-mail:kris.luyten@uhasselt.be
}

3D interaction may be the inhibitor which leads its users to experience frustration and poor performance as well.

To deal with user frustration in virtual environments, one prospective solution is supporting adaptive 3D user interfaces by providing adaptivity to users during their interaction in virtual environments. The design of adaptive user interfaces is considered key to improve user interaction with systems by facilitating user performance and helping users deal with complex systems [14]. Not much research has focused on investigating adaptive 3D user interfaces in virtual environments. In particular, little attention has been spent on providing adaptivity based on user frustration to assist users while interacting in virtual environments.

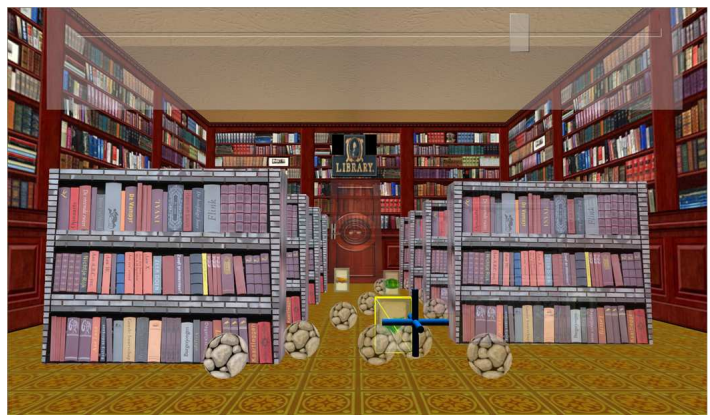

(a) Before adaptation: target object is occluded

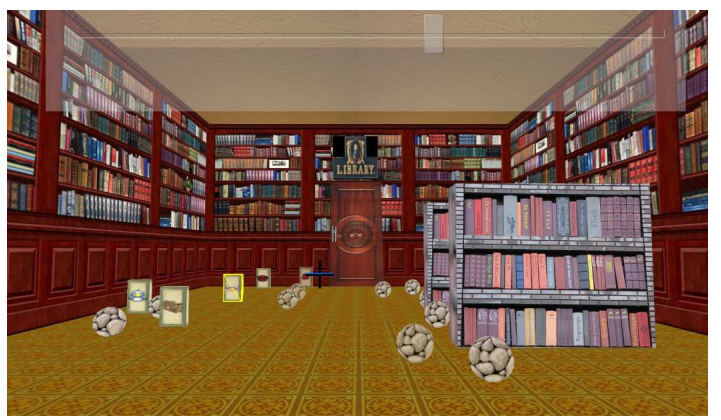

(b) After adaptation: occluded target becomes visible and highlighted

Figure 1: Example of adaptation in 3D environment

In this paper, we propose to provide adaptive assistance to users who are frustrated during their interaction with 3D user interfaces in virtual environments. The type of adaptation investigated is enhancing the 3D interaction with the use of multimodal feedback such as visual or force feedback. For instance when a user is detected to experience frustration, we can assist in performing object selection tasks by guiding him towards a predefined target object needed to be selected with the use of visual feedback. As shown in Figure 1(b), when adaptation takes place, the target object will be highlighted and the occluded target(s) will be made visible through 
a transparency feature. We realize that the usefulness of this type of adaptation may be limited to a situation where the system has knowledge of what the user's task is. For example, it might be useful in a gaming context where the user's selection goal is defined by the system and the system can control the difficulty of the selection based on the user's current frustration level. Our study aims to investigate the utilization of user frustration as trigger for providing run-time adaptation (i.e. adaptivity) in $3 \mathrm{D}$ virtual environments. This implies the establishment of a sort of monitoring module, which assesses and makes inferences about the state of user frustration based on the physiological signals measured during the interaction.

Measuring users' physiological signals has proven to be effective for determining user frustration [9, 23]. However, attaching users to the physiological sensor cables connected to the measuring device has been considered to be obtrusive and troublesome, especially if we intend to investigate user frustration continuously for a long period of time. Therefore, we would like to explore the possibility of utilizing other behavioral measures that might be promising to complement or even substitute the physiological measures in order to determine user frustration. This leads to the contribution of our paper, which is the investigation on the potential of determining user frustration based on the pressure patterns exerted on a 3D input device (i.e. Phantom stylus).

\section{Related Work}

Static interfaces, which behave in the same way regardless of the individual user, are considered less satisfactory in many cases since users have different characteristics (e.g. preferences, abilities, and levels of experience) that may influence their performance in using an interface. Adaptive user interfaces, which can be considered as a way to accommodate these individual differences, have gained a significant interest in the HCI research community for the past several years. Much research has focused on the windows, icon, menu and pointing (WIMP) applications [6, 22], while adaptive user interfaces for virtual environments has received only limited attention.

\subsection{Adaptation in 3D Virtual Environments}

One possible reason why adaptation in 3D virtual environments has been explored less often could be that interaction in 3D interfaces is more complex than in WIMP applications with $2 \mathrm{D}$ user interfaces. Nonetheless, there has been a number of research efforts in this direction. Wingrave et al. [28] investigated an approach to VE interface design, namely personalized nuance-oriented interaction, where the designer learns the user's preferred method of interaction and adapts the interaction to behave more like what the user wants. Hughes et al. [11] suggested adaptive navigation support for 3D virtual environments of e-commerce applications by directing customers' attention to significant features and integrating ideal viewing parameters with the navigation of a virtual environment. These works share the similar idea of supporting adaptation in 3D virtual environments by learning user characteristics. The first work focused on adaptivity to make the user interaction shorter and more preferable, and the latter focused on adaptivity to assist the user to work more efficiently by avoiding common problems encountered during the interaction.

The aforementioned works concentrate on providing adaptation by taking into account user preferences or interaction behavior. Dachselt et al. [4] took one step further by not only considering user preferences, but also device capabilities, user's location and other context information in their rigorous AMACONT adaptation architecture. Although many aspects have been taken into account in AMACONT, it may be also important to consider user frustration as another contextual information for adaptation in 3D virtual environments. Our previous work [20] has drawn on user frustration to investigate user's reaction concerning the adaptation of $3 \mathrm{D}$ interaction techniques in virtual environments and found that users experience less frustration when appropriate adaptations are made. However to our knowledge, no research to date has considered continuously monitoring user frustration and using this as a trigger for adaptations in the 3D environment. This has initiated our investigation on utilizing user frustration detected during the interaction in the $3 \mathrm{D}$ environment to drive the action of providing adaptation.

\subsection{Frustration-driven Adaptation}

Over a decade ago, Picard [21] initiated the research area of affective computing, which has been described as computing that relates to, arises from, or deliberately influences emotions. Ever since, her work has inspired a number of researchers to explore how computer systems may be designed to recognize, support and respond to user emotions (both negative emotion such as frustration or anger, and positive emotion such as happiness or joy). A number of works have addressed the design of interactive systems that support users to manage frustration.

Klein et al. [13] demonstrated an interactive-text based agent designed to help frustrating users by providing active emotional support to relieve their frustration. Gilleade and Dix [7] investigated the use of a player's frustration as a reference point to design adaptive video games for assisting problematic gameplay. Healey and Picard [10] presented a study of determining drivers' frustration to manage in-vehicle applications such as music selection and navigation aids for decreasing their frustration. So far, no work has investigated on how to manage frustration experienced by users during their interaction in $3 \mathrm{D}$ virtual environments.

Users have many different ways of expressing frustration, they may communicate frustration verbally (e.g. voice tones, verbalized statements) or physically (e.g. body postures, facial expressions). These determine the different ways of measuring user frustration such as self-reports [13], observations [7] and performance measures [3]. Besides behavioral changes, frustration also can be measured through physiological changes within the body of users. When people are frustrated, their heart races, their muscles tense and their palms become sweaty. Human's autonomic nervous system may respond to frustration by increasing skin conductance, increasing muscle tension or increasing heart rate [2].

Compared to highly subjective measures, using physiological signals has been thought to be more objective to measure user emotions [10, 29]. Mandryk et al. [17] and Scheirer et al. [23] have elaborately described their thorough investigations on the use of physiological sensors as input to infer the user's emotional state. Fairclough [5] extensively discussed the potential of physiological computing, which employs real-time physiological measures to infer users' psychological or emotional state as the basis for real-time system adaptation.

In this work, we aim to respond to user frustration by providing adaptive assistance with the expectation to improve user performance and interaction in 3D virtual environments. We make use of physiological signals to objectively measure user frustration. Thus we expect to assist users who experience frustration during their interaction in $3 \mathrm{D}$ virtual environments by using their state of being frustrated as a trigger to provide adaptation.

\subsection{Behavioral Indicators of Frustration}

One drawback in using physiological signals to measure frustration during user interaction is the inconvenience of doing so for a long duration or in a situation where the mobility of the user is necessary. Users might find the idea of being continuously attached to physiological sensor cables while they have to perform complex tasks, to be obtrusive and impractical.

Several studies have investigated the use of behavioral measures as indicators of user frustration, such as facial expressions, body postures or gestures, and pressure applied on devices, which are 
considered to be less obtrusive. Jaksic et al. [12] employed users' facial expression to indicate user frustration while browsing an online shopping website; a social agent is designed to respond and reduce user frustration. van den Hoogen [26] investigated the use of multiple behavioral measures, body postures and pressure on input devices (mouse and keyboard), as indicators of users' gameplay experience, including frustration. A few more studies have demonstrated that pressure exerted on other input devices, such as a touchpad [18] and buttons on a game console [25], can also be used to recognize user frustration. All of their findings suggested that the pressure applied on such input devices increases with user's frustrating experiences and difficulty levels in a game.

Most of the previously mentioned works examine the user state of being frustrated and determine the level of frustration based on subjective responses such as self-reported data or questionnaires. The users had to identify the critical incidents when they feel frustrated and rate their frustration on a five- or seven-point Likert-type scale. Based on this information, the behavioral measures were then analyzed to find a correlation with the self-reported measures with regard to frustration. In our approach, we propose to investigate the correlation between behavioral measures and the user frustration state, which is determined based on the user's physiological measures. As discussed before, using physiological signals to measure user frustration is considered to be more objective. The behavioral measure investigated in this study is the finger pressure exerted on a 3D input device, which to our knowledge no one has considered for this purpose before. By exploring these pressure patterns, we expect to utilize this measure as a behavioral indicator of frustration that may be potential as an alternative to using physiological measures.

\section{Detecting user frustration}

Our goal in the presented research is to objectively measure the level of frustration of users during their interaction in 3D virtual environments and respond to it by providing adaptation whenever user frustration is detected. Frustration discovery is not only enabled by physiological measures, but we also investigate the potential of detecting this user state by using behavioral measures, which is the finger pressure exerted on a 3D input device in our case.

Several physiological measures have demonstrated to be effective in determining user frustration [2]: facial muscle activity or Electromyography (EMG), skin conductance or Galvanic skin response (GSR), blood volume pulse (BVP), heart rate (HR) and respiration rate $[9,10,17,23]$. Based on these previous works, we chose to collect three physiological signals: EMG, GSR and BVP (to derive HR measures later on) to measure user frustration in our work. We utilized the ProComp Infiniti hardware ${ }^{1}$ and $\mathrm{Bi}$ ograph software, a device for real-time computerized biofeedback and physiological data acquisition, as shown in Figure 2.

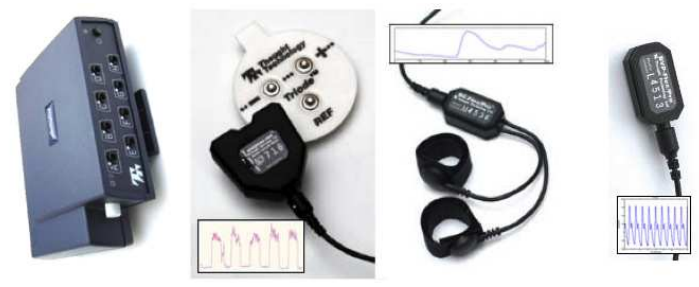

Figure 2: ProComp Infiniti encoder and the physiological sensors: EMG, GSR, BVP (from left to right).

During their interaction in 3D virtual environments, users were attached to the physiological sensors, which pass the captured sig-

${ }^{1}$ http://www.thoughttechnology.com

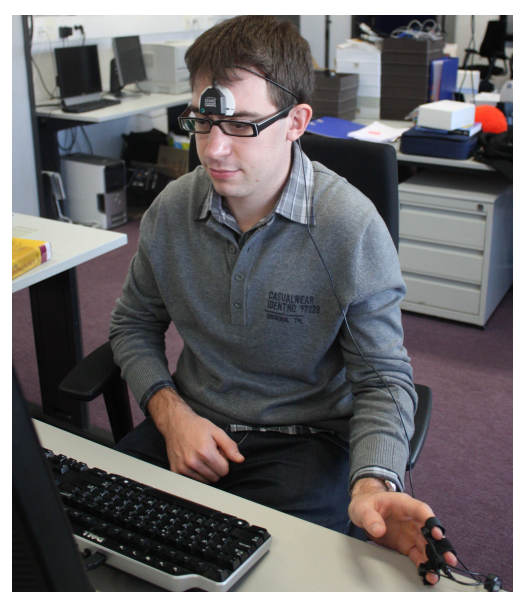

Figure 3: One participant attached to the three physiological sensors used in this study.

nals to a computer via the microprocessor-controlled ProComp Infiniti encoder, as illustrated in Figure 3. To measure the facial muscle activity, the Surface Electromyography sensor (MyoScan-Pro 400) was placed on the forehead of users near to the eyebrow. The other two sensors for measuring skin conductance and blood volume pulse were fixed on the idle hand of users (not used during the interaction). The Skin Conductance sensor (SC-Flex/Pro) was placed on the palmar surface of the index finger and the ring finger, while the Blood Volume Pulse sensor (BVP-Flex/Pro) was placed by pressing it against the palmar surface of the middle finger.

Our approach to deduce the emotional state is inspired by Mandryk [16]. In her research, a fuzzy logic approach was used to develop two models to determine user emotions: the first model transforms four physiological signals $\left(\mathrm{EMG}_{\text {frown }}, \mathrm{EMG}_{\text {smile }}, \mathrm{GSR}\right.$, HR) into arousal and valence, and the second model transforms arousal and valence into five emotions: frustration, boredom, challenge, excitement and fun. Arousal is described as a state of heightened physiological activity and valence describes where an emotional reaction sits on an axis from the positive to the negative. Based on these models, a number of rules have been established to enable the transformation of physiological signals into five emotional states by means of arousal and valence [16]. For this study, we adopted the rules to transform three physiological signals $\left(\mathrm{EMG}_{\text {frown }}, \mathrm{GSR}, \mathrm{HR}\right)$ into frustration as follows.

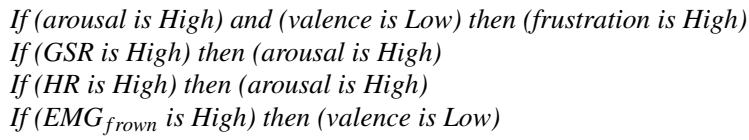

To determine the user frustration state, first we collected the raw data of $\mathrm{EMG}_{\text {frown }}, \mathrm{BVP}$, and GSR. These captured signals were digitized, encoded and transmitted to a computer running the Biograph software. EMG data were collected at $2048 \mathrm{~Hz}$, while BVP and GSR were collected at $256 \mathrm{~Hz}$. To remove unwanted noise and other unnecessary frequencies from the data, it is important to filter the raw data of the physiological signals being collected [24]. With the help of Biograph software, we filtered the raw data using the Infinite Impulse Response (IIR) filter. For EMG and BVP, we filtered the data with a Butterworth band pass filter at a defined low and high cutoff frequency (EMG at $0.8 \mathrm{~Hz}, 2.5 \mathrm{~Hz} ; \mathrm{BVP}$ at $0.2 \mathrm{~Hz}$ and $2.2 \mathrm{~Hz}$ ). For GSR, we used a Butterworth low pass filter at $0.6 \mathrm{~Hz}$. After filtering the BVP signals, we derived the measure of Heart Rate (HR) by computing the beat-to-beat inter-beat interval of the signals. 
Since EMG and BVP/HR are bipolar signals, we conducted a rectification process to convert them to monopolar signals (the positive and negative signals were converted into all positive values). For this purpose, we used the Root Mean Square (RMS) sliding window technique and smoothed the data with an averaging factor of 128 over a time period of 0.125 seconds. Then, we calculated the running mean of the computed EMG, BVP/HR, and GSR signals. We used these mean values as inputs to our designed application of a 3D virtual environment. The Biograph software is supplemented with the Connection Instrument, which gives the possibility to connect a computer running Biograph to third-party applications utilizing the measured signals.

In order to realize real-time detection of user frustration, we established a monitoring module that assesses and makes inferences about the state of user frustration based on the physiological signals measured during the interaction. This monitoring module takes input from the Biograph software and performs a decision-making process to determine whether a user is frustrated or not in realtime. The assessment of user frustration is made with reference to absolute criteria [5], usually referred to as the baseline. The baseline measures the resting level of an activity of the physiological measures prior to simulation. In our study, we measured the baseline and made the assessment of frustration with reference to it (the physiological measures are considered to be high when they exceed $5 \%$ of the normal baseline [15]). The decision-making rules to determine the detection of user frustration are expressed as simple If/Then statements as follows.

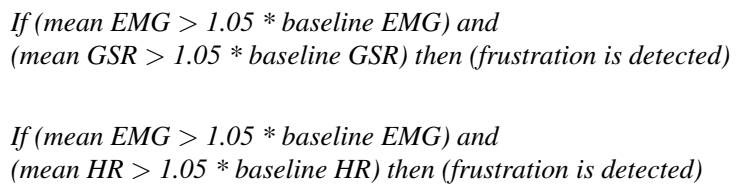

Through the monitoring module, it is possible to detect in realtime user frustration in 3D virtual environments based on user's physiological measures. Whenever user frustration is detected, adaptive assistance is provided to the users as response to their frustration. Furthermore, we will investigate the potential of detecting user frustration in 3D virtual environments through the measures of finger pressure exerted on the 3D input device.

\section{EXPERIMENT}

Before describing the user experiment, we recapitulate our research objective. The goal is to provide adaptive assistance to users based on their frustration detected during the interaction with 3D user interfaces in virtual environments. To deal with the obtrusiveness of using physiological sensors to detect frustration, we investigate the possibility of detecting user frustration based on the pressure patterns exerted on a 3D input device. For this purpose, an experiment was conducted with a controlled setting of a virtual environment where participants perform object selection tasks to acquire certain targets. Object selection is a fundamental task in virtual environments and any interactive 3D user interfaces must support performing this task [27].

\subsection{Context}

In this study, we focused on investigating user interaction when performing a 3D target acquisition task in a virtual environment, where users are required to search and select certain objects. As shown in Figure 4, the scene of the experiment resembles a library environment, where several objects are scattered around the room and are sometimes hidden behind the bookshelves. For each target object to be found, a clue was given beforehand as a brief description of the object's attributes. To select the target objects, we employed the bubble cursor technique [8] since a former study proved that this selection technique performs better than the others when selecting objects in dense and occluded virtual environments [27].

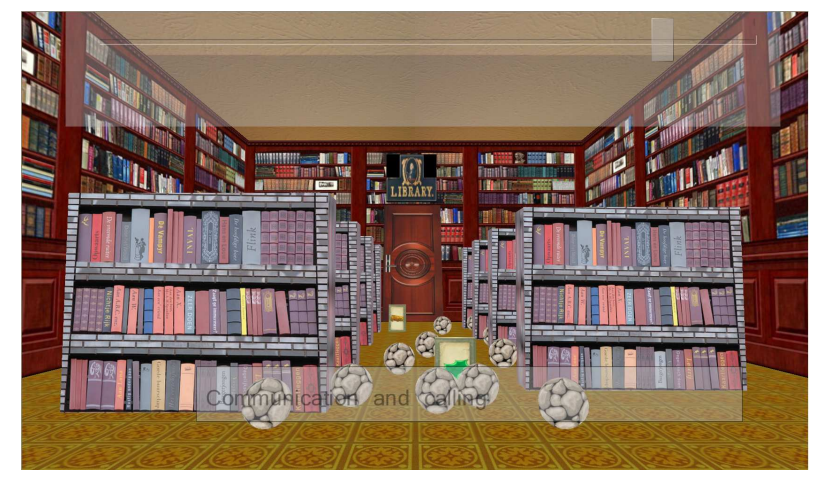

Figure 4: The experiment environment.

Once user frustration is detected while interacting in the virtual library, we aim to enhance the 3D interaction of users by providing adaptive assistance through the incorporation of feedback, namely visual feedback, haptic feedback and multimodal feedback (visual and haptic). The integrated feedback aims to provide assistance by guiding the users towards a target object to be selected. The visual feedback takes the form of highlighting the target object and adding transparency to make the occluded target visible, while the form of the haptic feedback is giving a drawing force towards the target object. The feedback is designed to be temporary (only lasts for 5 seconds) so the given assistance somewhat acts like an extra hint for the users to perform the task more efficiently.

\subsection{Methods}

\subsubsection{Participants}

Sixteen participants (13 males and 3 females) ranging between 21 and 35 years old were recruited to take part in the experiment. A large number of participants were people with a computer science background, most of them have previously experienced interacting with 3D user interfaces in virtual environments (only 1 participant was a first-timer). Only six participants have prior experience in performing object selection tasks with the bubble cursor technique, while the rest have never used this selection technique. All of them were right-handed and used their dominant hand to operate the device.

\subsubsection{Apparatus}

We used two desktop computers (2.33 Ghz, 1.96 Gb RAM) connected over a LAN, one computer is used for running the $3 \mathrm{D}$ environment (19" monitor at $1680 \times 1050$ resolution) and the other one is for running the Biograph software. As input device, a Phantom Premium 1.0 haptic device with stylus was used. Since we intend to capture the finger pressure measures exerted on the input device, we embedded the Phantom stylus with two pressure sensors, FlexiForce Sensor Model A201 (6" length with force ranges of 0-25 lbs), as shown in Figure 5(a). As previously indicated in Section 3, we utilized a physiological measuring device, the ProComp Infiniti encoder SA7500 and the Biograph Infiniti software version 5.0.3, with three physiological sensors (EMG, GSR and BVP) attached to the fingers of participants. Figure 5(b) illustrates the setup of the experiment apparatus.

\subsubsection{Procedure}

The experiment consisted of four sessions, each session was represented with a different scene (such as the one in Figure 4). Participants were asked to execute a target acquisition task based on 


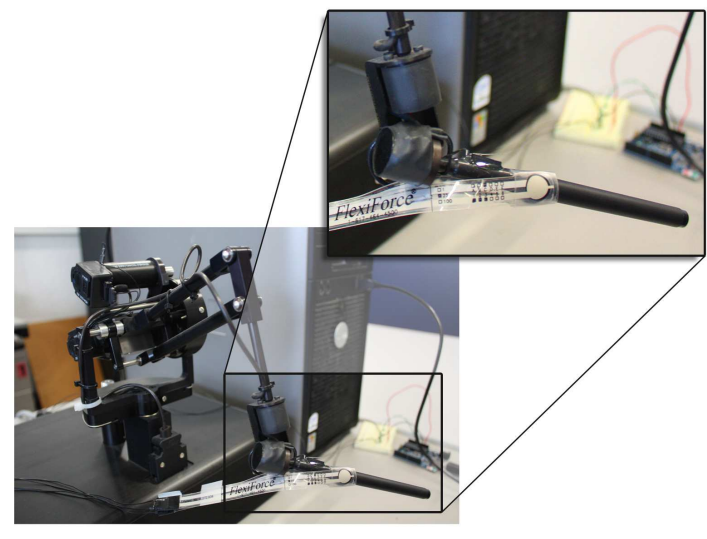

(a) The Phantom stylus embedded with pressure sensors

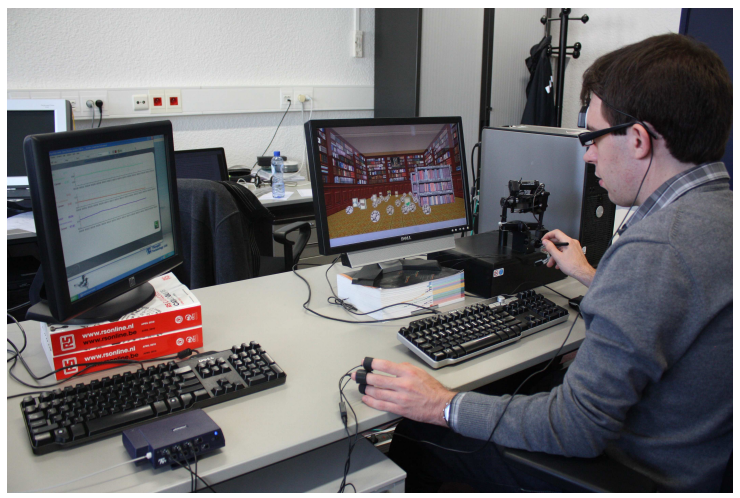

(b) The experiment setting

Figure 5: The experiment apparatus.

the given clues. They had to search for the target objects as fast as possible and then select the targets when found. For each scene, participants were required to acquire five targets within the given maximum time of 3 minutes. We continuously captured and transferred the physiological signals of the participants during the experiment sessions into the Biograph software to enable detecting frustration and providing adaptation when necessary.

Before the experiment, participants were given a practice session to help them get acquainted with the $3 \mathrm{D}$ environment and the Phantom device. At the beginning of each session, we measured the physiological baseline of the participants for 3 minutes. During the baseline measurement, participants were asked to relax as much as possible while watching a relaxing aquatic movie clip. To determine individuals' pressure indexes, after the baseline measurement of the first session, participants were asked to hold the Phantom stylus as hard as they can (but still in a natural and comfortable way) for 30 seconds. This was done to acquire the maximum pressure value that one user is likely to exert. After each session of the experiment, participants completed a post-experiment questionnaire regarding their experience when interacting in the 3D environment with regard to the level of frustration and difficulty they experienced. Averagely, the experiment lasted for about 45 minutes per participant.

\subsubsection{Design}

A within-subjects (repeated measures) design was used in this experiment. The independent variable was the condition of adaptation with four levels: no adaptation, adaptive assistance with visual feedback, adaptive assistance with haptic feedback, and adaptive assistance with multimodal feedback. To deal with carryover effects, the order of these conditions was counterbalanced across the participants using a 4 x 4 balanced Latin square. We measured task completion time and pressure index as dependent variables.

\section{Results and Discussion}

In this study, we have established a monitoring module that enables continuous observation of user's physiological measures and determines in real-time whether the user is frustrated or not. In this monitoring module, user frustration is recognized and monitored as a trigger for providing adaptation. In our experiment, we put the monitoring module in practice and analyzed the patterns of pressure that users exerted on the Phantom stylus as a possible way to detect user frustration more unobtrusively. In addition to that, we also investigated the effectiveness of adaptation provided as a result of user frustration.

\subsection{Pressure Exertion on Input Device with respect to User Frustration}

To achieve detecting user frustration in a more unobtrusive manner, we investigated the pressure measure that users exerted on a 3D input device and its relation with user frustration. Previous studies have indicated that the pressure applied on input devices increases with user's frustrating experiences $[18,25,26]$. We believe that the finger pressure exerted on the Phantom stylus will increase when user frustration is detected. Our hypothesis is that participants will exert higher pressure when they are frustrated.

From the raw data collected by the pressure sensors, we calculated pressure indexes using a linear interpolation approach. We interpolated every pressure value measured into the range of pressure values, to determine a pressure index between 0 and 1 . We distinguished the data into two categories, the pressure indexes when the user is frustrated and when the user is not frustrated. The distinction of the two conditions, frustrated or not frustrated, is determined by the physiological measures (see the algorithms described in Section 3). One-way ANOVA showed that there is a significant difference of Pressure index $\left(F_{1,319}=20.68, \mathrm{p}<0.001\right)$ between the frustrated and not frustrated condition. Average pressure indexes were $0.397(\mathrm{SD}=0.017)$ for condition when the user is frustrated and $0.296(\mathrm{SD}=0.018)$ for condition when the user is not frustrated. This finding showed that the pressure exerted by participants on the Phantom stylus were significantly higher when they were frustrated than when they were not. In conclusion, when users are frustrated, they exert more pressure on the input device.

We also investigated the relationship between the physiological measures and the pressure measures. From the collected physiological data, we derived the physiological indexes that represent a fraction of the measured physiological data from the baseline (see Section 3). Based on Pearson's correlation tests, we found significant positive correlations of the physiological indexes (EMG, GSR, and HR) with the pressure index ( $r=0.467$ for EMG, $r=0.655$ for GSR, $r=0.573$ for HR, $\mathrm{p}<0.001)$. The measures of pressure are highly correlated with the measures of physiological signals. The finding suggested that as the participant's physiological measures increase, the pressure exerted by participants on the Phantom stylus increases as well. In another way, we can say that the more frustrated users are, the more amount of pressure they exert on the input device.

We would like to further examine the possibility of substituting the physiological measures with the pressure measures to determine user frustration. For this purpose, we built two predictive models of frustration using linear discriminant analysis: the first 
model used the physiological indexes (EMG, GSR, and HR) as the predictors and the second model based the prediction on the pressure index. The results of the discriminant analysis showed that all of the discriminant functions have significant Wilks Lambda (0.876 for EMG, 0.731 for GSR, 0.623 for HR, 0.438 for Pressure, $\mathrm{p}<0.001)$. For the first model, the overall classification accuracy is $83.3 \%$ (65.8\% for frustrated and $100 \%$ for not frustrated). With the second model, the accuracy decreases to $81.3 \%(79.5 \%$ for frustrated and $83.1 \%$ for not frustrated). These findings suggested that $81.3 \%$ of the time, inferring frustration based on the pressure measures is the same (i.e. has the same accuracy) as inferring it based on the combined physiological measures of EMG, GSR and HR. Only $2 \%$ of accuracy is lost when inferring frustration by using the pressure sensors alone. Based on this, the idea of utilizing the finger pressure measures as a behavioral indicator of frustration as an alternative to using physiological measures shows a great potential. More research is necessary to enable inferring user's frustration state with its severity based on the pressure they exert on an input device. In our experiment to infer user frustration based on the physical pressure exerted by users on a 3D input device, we want to verify whether certain patterns of users' finger pressure exist.

\subsection{Exploratory Investigation of Pressure Patterns}

To investigate the pressure patterns, we looked at the trends of pressure values measured (described by pressure index) during their interaction in a virtual environment. During the experiment, we observed three kinds of situations encountered by users with regard to frustrating experiences. First, a user is never frustrated from the beginning until the end of the interaction. Second, a user always experiences frustration during the whole interaction. And the last situation is where a user only starts to get frustrated in the middle of the interaction, with a possibility that the frustration stops during the interaction.

Exactly half of the participants experienced all of the aforementioned situations in the experiment. Although in different orders, 8 participants experienced being never frustrated, always frustrated and partially frustrated, in 3 out of 4 sessions of the experiment. Figure 6(a) and Figure 6(b) illustrate the pressure indexes of participants for the situation of them being never frustrated and always frustrated during the whole interaction. As we can see, both situations showed a tendency of having approximately flat lines without any pronounced peaks or depressions of pressure indexes. The main observed difference was the values of pressure indexes between the two situations. When always frustrated, participants' pressure indexes were higher (ranging from 0.2 to 0.4 ) compared to when they were never frustrated (between 0.05 and 0.2 ). We can also observe that when participants were always frustrated, the task completion times were faster due to the adaptation provided when frustration was detected.

Another situation was where participants were partially frustrated. The participants started with a state of being not frustrated, but then began to experience frustration during the experiment session after few seconds of interaction. As shown in Figure 6(c), pronounced changes of pressure indexes can be observed. At the moment participants got frustrated, a significant increase of pressure index occurred until it became stagnant. In a few cases, the participants experienced a decrease of frustration shown by the decreasing of the pressure indexes. Two participants (Participant 1 and 8) even reached the initial point, which means that they returned to the state of being not frustrated.

Based on our data, we can deduce that when a user is at one distinctive state, either frustrated or not frustrated, the pressure indexes form a flat trend line. When a change of state occurs, for example the user becomes frustrated from being not frustrated, the pressure indexes break the flat trend line and a significant change (either an increment or a decrement) can be observed. Figure 6(d) gives a brief overview of this observation.

\subsection{User's Performance with respect to Adaptation}

When users are frustrated, it is most likely that they will experience a significant decrease in their performance [3]. However adaptation has been considered as a solution to improve user interaction in complex interactive systems by facilitating user performance [14]. We believe that the performance of frustrating users in virtual environments will increase when adaptation is provided. Therefore, we hypothesized that participants will perform faster when adaptive assistance is provided.

We analyzed four conditions of adaptation in this experiment: (1) no adaptation, (2) adaptive assistance with visual feedback, (3) adaptive assistance with haptic feedback, and (4) adaptive assistance with multimodal feedback. Repeated measures analysis of variance (RM ANOVA) showed a significant main effect of Condition on Task completion time $\left(F_{3,45}=5.09, \mathrm{p}<0.005\right)$. This indicates a significant difference between the four conditions. Average task completion times were $22.14 \mathrm{~s}(\mathrm{SD}=2.22)$ for condition of no adaptation, $20.33 \mathrm{~s}(\mathrm{SD}=2.68)$ for condition of adaptive assistance with visual feedback, $13.15 \mathrm{~s}(\mathrm{SD}=1.89)$ for condition of adaptive assistance with haptic feedback, and $12.53 \mathrm{~s}(\mathrm{SD}=1.7)$ for condition of adaptive assistance with multimodal feedback. Tukey's HSD post hoc comparisons showed that both conditions of adaptive assistance with haptic feedback and multimodal feedback were significantly faster than the condition of no adaptation and adaptive assistance with visual feedback $(\mathrm{p}<0.05)$. However, the condition of adaptive assistance with visual feedback was not significantly faster than the condition of no adaptation $(\mathrm{p}=0.6)$. Moreover, the condition of adaptive assistance with multimodal feedback was not significantly faster than the condition of adaptive assistance with haptic feedback $(\mathrm{p}=0.79)$.

Based on these findings, we discovered that providing adaptation increases user performance since participants performed faster when adaptive assistance was provided. The insignificant difference found between the conditions of no adaptation and adaptive assistance with visual feedback, suggests that the implemented visual feedback may not be appropriate for the users. We suspected that the action of highlighting and adding transparency was not successful to capture the attention of participants towards the target object needed to be selected. Some participants agreed that the visual feedback could have helped them to focus on where to look. However, they failed to notice the visual feedback since they were looking and paying attention to the other part in the environment. One participant remarked that making the target object to blink or flicker might help him better.

A significant increase of performance was only shown when adaptive assistance was provided through the incorporation of haptic and multimodal feedback (visual and haptic combined altogether). However, no significant difference was observed between them. This is not surprising when taking into account the fact that our visual feedback was not that effective. The multimodal feedback might have better results when the haptic feedback is combined with a better designed visual feedback. From these findings, we can conclude that providing adaptation in haptic interaction based on the frustration of the user results in a better overall performance in this particular interaction scenario.

\subsection{User's Subjective Ratings on Adaptation}

In the experiment, we acquired the subjective responses of participants concerning their opinion with respect to the level of frustration and difficulty they experienced in the four conditions of adaptation. After each session of the experiment, participants were asked to complete a questionnaire and indicate their responses on a 5point scale rating (e.g. 1 not frustrated at all to 5 very frustrated). 


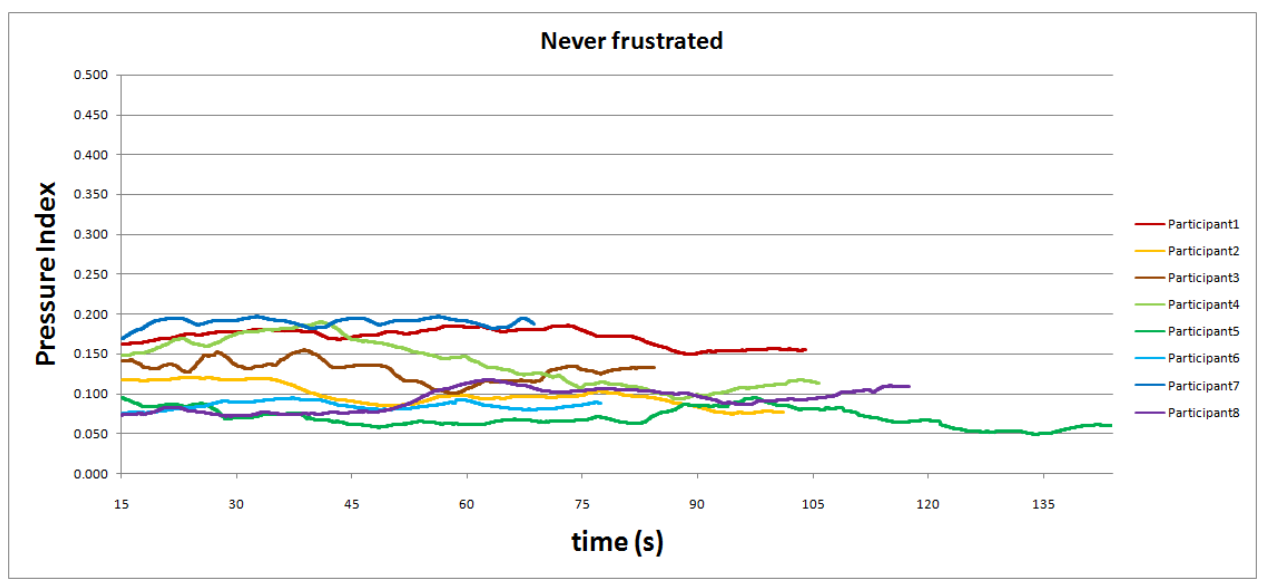

(a) Participants were never frustrated

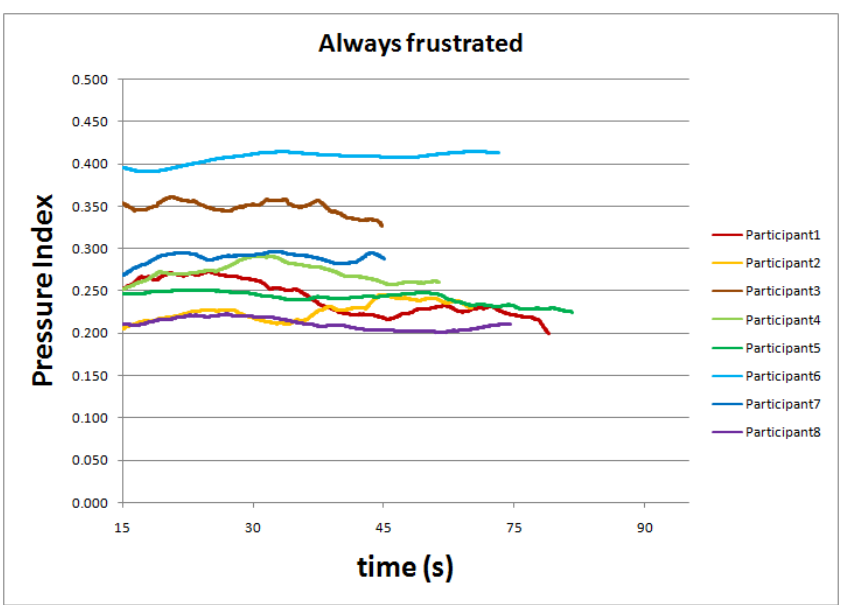

(b) Participants were always frustrated

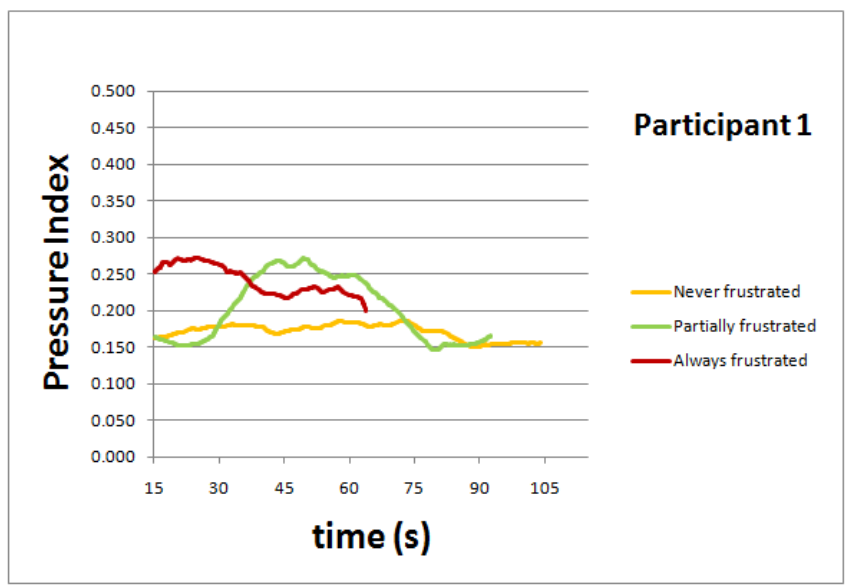

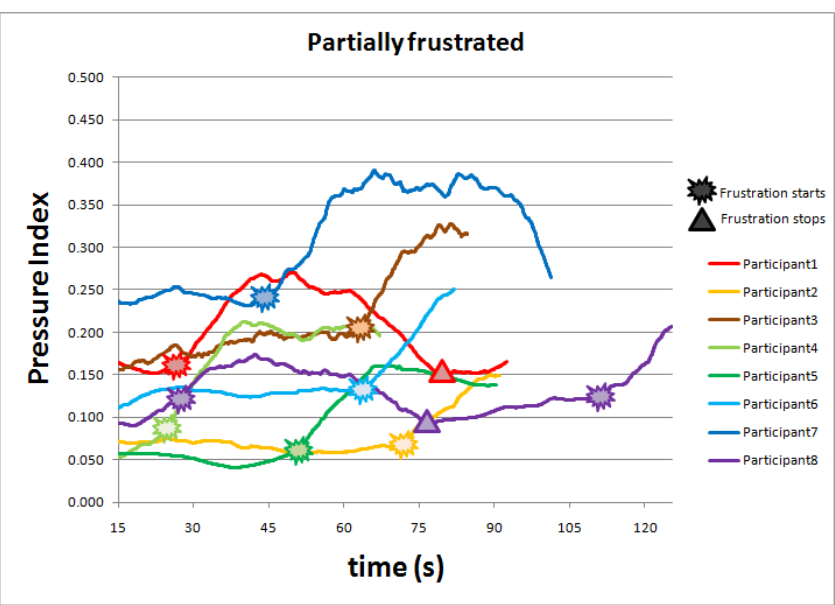

(c) Participants were partially frustrated

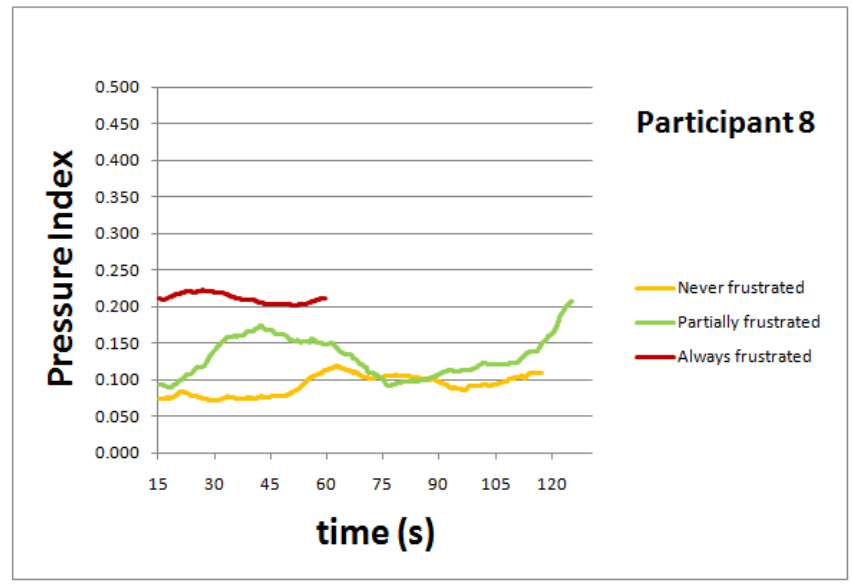

(d) Overview of the trends observed on two participants

Figure 6: Pressure patterns (shown timeline is concatenated for five target acquisition tasks of the whole session). 
Based on the subjective ratings, we found that the average ratings for conditions where adaptive assistance is provided was higher than the no adaptation condition. Concerning the level of frustration, participants felt the most frustrated in the condition of no adaptation (3.44) and the least in the condition of adaptive assistance with haptic feedback (2.19). For the other conditions, adaptive assistance with visual feedback and multimodal feedback, the average ratings were 2.63 and 2.44 respectively. Regarding the difficulty level, participants perceived the condition of no adaptation to be most difficult (3.06) and the condition of adaptive assistance with haptic feedback to be least difficult (2.56). The average ratings were 2.88 and 2.63 for the conditions of adaptive assistance with visual feedback and multimodal feedback respectively. This finding confirms that users perceive themselves to undergo less frustration and perform the task less difficult when the investigated adaptation is incorporated.

\section{Conclusion}

In this paper, we have discussed our work of supporting adaptivity to users for dealing with their frustration during interaction in virtual environments. We established a monitoring module that continuously captures the physiological measures of users and detected in real-time when frustration takes place. When user frustration is recognized, it triggers an action of providing adaptive assistance to users through the incorporation of feedback (visual, haptic and multimodal). The paper contributes with the investigation of the possibility of detecting user frustration based on the pressure exerted by users on a 3D input device. We observed that a significantly higher pressure is exerted on the input device as users experience frustration, and a linear relationship is shown between the measures of exerted pressure and the severity of frustration. We also presented an overview of the pressure patterns observed with regard to the user frustration states.

An experiment was conducted to investigate the effect of adaptation provided as a response to the detected user frustration. Our findings confirm that providing adaptation resulted in a significant increase of user's performance in this 3D interaction scenario, but only through the adaptation of incorporating haptic feedback. Besides increased performance, another advantage of adaptation is that users discover themselves to feel less frustrated and experience less difficulty in performing the tasks. However, we are aware that the effectiveness of the adaptation as such is not proven. Follow up research is planned to study the effect of the initial situation, the personalization of the adaptation and the recurring adaptation, such as switching back and forth between types of adaptations.

\section{ACKNOWLEDGEMENTS}

This research was partly funded through the INTERREG-IV program (project IVA-VLANED-1.14, Euregio Benelux). The authors would like to acknowledge the researchers involved in this project, especially Lode Vanacken and Tom De Weyer for their assistance in the implementation work for the experiment. We also thank the participants for their valuable contributions in the experiment.

\section{REFERENCES}

[1] D. A. Bowman, J. Chen, C. A. Wingrave, J. Lucas, A. Ray, N. F. Polys, Q. Li, Y. Haciahmetoglu, J.-S. Kim, S. Kim, R. Boehringer, and T. Ni. New directions in 3d user interfaces. Int. J. Virtual Reality, 5(2):3-14, 2006.

[2] J. T. Cacioppo, L. G. Tassinary, and G. Berntson. Handbook of Psychophysiology. Cambridge University Press, 2007.

[3] I. Ceaparu, J. Lazar, K. Bessiere, J. Robinson, and B. Shneiderman. Determining causes and severity of end-user frustration. Int. J. of Hum.-Comput. Interact., 17:333-356, 2004.

[4] R. Dachselt, M. Hinz, and S. Pietschmann. Using the amacont architecture for flexible adaptation of $3 \mathrm{~d}$ web applications. In Web3D '06, pages 75-84, New York, USA, 2006. ACM.
[5] S. H. Fairclough. Fundamentals of physiological computing. Interact. Comput., 21(1-2):133-145, 2009.

[6] K. Gajos and D. S. Weld. Supple: automatically generating user interfaces. In IUI '04, pages 93-100, New York, USA, 2004. ACM.

[7] K. M. Gilleade and A. Dix. Using frustration in the design of adaptive videogames. In $A C E^{\prime}$ '04, pages 228-232, New York, USA, 2004. ACM.

[8] T. Grossman and R. Balakrishnan. The bubble cursor: enhancing target acquisition by dynamic resizing of the cursor's activation area. In CHI '05, pages 281-290, New York, USA, 2005. ACM.

[9] R. Hazlett. Measurement of user frustration: a biologic approach. In Extended abstracts CHI '03, pages 734-735, New York, USA, 2003. ACM.

[10] J. Healey and R. W. Picard. Detecting stress during real-world driving tasks using physiological sensors. IEEE Transactions on Intelligent Transportation Systems, 6(2):156-166, 2005.

[11] S. Hughes, P. Brusilovsky, and M. Lewis. Adaptive navigation support in 3d e-commerce activities. In Workshop proceedings on Recommendation and Personalization in E-Commerce, pages 132-139, 2002.

[12] N. Jaksic, P. Branco, P. Stephenson, and L. M. Encarnaç ao. The effectiveness of social agents in reducing user frustration. In Extended abstracts CHI '06, pages 917-922, New York, USA, 2006. ACM.

[13] J. Klein, Y. Moon, and R. W. Picard. This computer responds to user frustration: Theory, design, and results. Interact. Comput., 14(2):119 $-140,2002$.

[14] T. Lavie and J. Meyer. Benefits and costs of adaptive user interfaces. Int. J. Hum.-Comput. Stud., 68(8):508-524, 2010.

[15] T. Lin, M. Omata, W. Hu, and A. Imamiya. Do physiological data relate to traditional usability indexes? In $\mathrm{OZCHI}$ '05, pages 1-10, Narrabundah, Australia, 2005. CHISIG of Australia.

[16] R. L. Mandryk. Modeling User Emotion in Interactive Play Environments: A Fuzzy Physiological Approach. PhD thesis, Simon Fraser University, Canada, 2005.

[17] R. L. Mandryk, K. M. Inkpen, and T. W. Calvert. Using psychophysiological techniques to measure user experience with entertainment technologies. Behaviour \& IT, 25(2):141-158, 2006.

[18] H. M. Mentis and G. K. Gay. Using touchpad pressure to detect negative affect. In ICMI '02, page 406, Washington, DC, USA, 2002. IEEE Computer Society.

[19] D. Norman. The design of everyday things. Doubleday, 1990.

[20] J. Octavia, C. Raymaekers, and K. Coninx. Adaptation in virtual environments: conceptual framework and user models. Multimedia Tools and Applications, pages 1-22, 2010. 10.1007/s11042-010-0525-z.

[21] R. W. Picard. Affective Computing. PhD thesis, MIT Press, Cambridge, MA, USA, 2008.

[22] C. Rocchi, I. Graziola, D. Goren-Bar, O. Stock, and M. Zancanaro. Adaptive multimedia guide. In PEACH: Intelligent Interfaces for Museum Visits, pages 3-22. Springer-Verlag New York, Inc., 2007.

[23] J. Scheirer, R. Fernandez, J. Klein, and R. W. Picard. Frustrating the user on purpose: a step toward building an affective computer. Interact. Comput., 14(2):93 - 118, 2002.

[24] R. M. Stern, W. J. Ray, and K. S. Quigley. Psychophysiological Recording. Oxford University Press, 2001.

[25] J. Sykes and S. Brown. Affective gaming: measuring emotion through the gamepad. In Extended abstracts CHI '03, pages 732-733, New York, USA, 2003. ACM.

[26] W. van den Hoogen, W. IJsselsteijn, and Y. de Kort. Exploring behavioral expressions of player experience in digital games. In A. Nijholt and R. Poppe, editors, Workshop proceedings on Facial and Bodily Expression for Control and Adaptation of Games, pages 11-19, 2008.

[27] L. Vanacken, T. Grossman, and K. Coninx. Multimodal selection techniques for dense and occluded $3 \mathrm{~d}$ virtual environments. Int. J. Hum.Comput. Stud., 67(3):237-255, 2009.

[28] C. A. Wingrave, D. A. Bowman, and N. Ramakrishnan. Towards preferences in virtual environment interfaces. In $E G V E$ ' 02 , pages 63-72, Aire-la-Ville, Switzerland, 2002. Eurographics Association.

[29] C. Yun, D. Shastri, I. Pavlidis, and Z. Deng. O' game, can you feel my frustration?: Improving user's gaming experience via stresscam. In CHI '09, pages 2195-2204, New York, USA, 2009. ACM. 